\title{
Ecuación dinámica para estimar el crecimiento en diámetro de Pinus montezumae Lamb. en Puebla, México
}

\author{
Dynamic equation to estimate the growth in diameter of Pinus \\ montezumae Lamb. in Puebla, Mexico \\ Juan Carlos Tamarit-Urias*, Gerónimo Quiñonez-Barraza², Xavier García-Cuevas³, \\ Jonathan Hernández-Ramos ${ }^{3}$ y José Carlos Monárrez-González ${ }^{2}$
}

\begin{abstract}
1 Instituto Nacional de Investigaciones Forestales, Agrícolas y Pecuarias. Centro de Investigación Regional Golfo-Centro. Campo Experimental San Martinito. Tlahuapan, Puebla, México
\end{abstract}

\begin{abstract}
2 Instituto Nacional de Investigaciones Forestales, Agrícolas y Pecuarias. Centro de Investigación Regional Norte-Centro. Campo Experimental Valle del Guadiana. Durango, Durango, México.
\end{abstract}

\author{
3 Instituto Nacional de Investigaciones Forestales, \\ Agrícolas y Pecuarias. Centro de Investigación \\ Regional Sureste. Campo Experimental Chetumal. \\ Chetumal, Quintana Roo, México. \\ * Autor de correspondencia. tamarit.juan@inifap.gob.mx
}

\section{RESUMEN}

El diámetro normal tiene correlación alta con otros atributos del árbol y del rodal, por tanto, su modelación es relevante. El objetivo de este trabajo fue desarrollar una ecuación dinámica para estimar, en función de la edad, el crecimiento e incremento en diámetro normal de árboles de Pinus montezumae. Se utilizó una muestra de 81 árboles colectados en la región "Ixta-Popo" de Puebla, México y se aplicó la técnica de análisis trocal. Se evaluó la calidad de ajuste de seis ecuaciones dinámicas expresadas en diferencia algebraica (DA) y en su generalización (DAG). La mejor ecuación fue seleccionada usando criterios estadísticos y análisis gráficos, esta se basó en un modelo de crecimiento de Korf expresado en DAG, que posteriormente se ajustó con el método de variables artificiales y se corrigió la autocorrelación y la heterocedasticidad. Esta ecuación presentó alta precisión y exactitud $(\mathrm{RCME}=2.393 \mathrm{~cm}$, sesgo $=0.055 \mathrm{~cm}) ;$ se usó para construir una familia de curvas de crecimiento con base en el índice de diámetro normal (IDn) para determinar el crecimiento corriente y medio anual (ICA e IMA), así como el tiempo de paso (TP) por categoría diamétrica (CD). Para una condición promedio (IDn $=37 \mathrm{~cm}$ ), el ICA máx fue de $1 \mathrm{~cm}^{2}$ año ${ }^{-1}$ que corresponde a $9.33 \mathrm{~cm}$ de Dn y se alcanza a los 14.8 años; el IMA máx fue de $0.79 \mathrm{~cm}$ año${ }^{1}$ que corresponde a $25 \mathrm{~cm}$ y sucede a la edad de 31.6 años; el TP promedio fue de 8.09 años para un intervalo de $5 \mathrm{~cm}$ a $55 \mathrm{~cm}$ con CD de $5 \mathrm{~cm}$. La ecuación desarrollada puede formar parte de sistemas de crecimiento y rendimiento maderable para el manejo sustentable de esta especie en la zona de estudio.

PALABRAS CLAVE: análisis troncal, diámetro normal, enfoque DAG, ICA-IMA, modelo de Korf, tiempo de paso.

\section{ABSTRACT}

The diameter at breast height (DBH) has a high correlation with other attributes of the tree and the stand, therefore its modeling is relevant. The objective was to develop a dynamic equation to estimate the growth and increment in DBH of Pinus montezumae trees as a function of age. A sample of 81 trees collected in the "Ixta-Popo" region of Puebla, Mexico was used, and the stem analysis technique was applied. The fit quality of six dynamic equations expressed in algebraic difference (DA) and generalized algebraic difference approach (GADA) was evaluated. The best dynamic equation was selected using statistical criteria and graphic analysis, this is based on the Korf growth model expressed in GADA, which was later adjusted using the artificial variables method, autocorrelation and heteroscedasticity were corrected. This equation presented high precision and accuracy (RMSE $=2.393 \mathrm{~cm}$, Bias $=0.055 \mathrm{~cm}$ ), it was used to construct a family of growth curves based on the DBH index (IDBH), to determine the current and average annual growth (ICA and IMA), as well as the passage times (PT) by diametric category (DC). For the average condition (IDBH $=37 \mathrm{~cm}$ ), the $\mathrm{CAI}_{\max }$ was $1 \mathrm{~cm}$ year ${ }^{-1}$ which corresponds to $9.33 \mathrm{~cm}$ of $\mathrm{DBH}$ and is reached at 14.8 years. The $\mathrm{MAI}_{\max }$ was $0.79 \mathrm{~cm}_{\text {year }}{ }^{-1}$, which corresponds to $25 \mathrm{~cm}$ and occurs at the age of 31.6 years; the average PT was 8.09 years for an interval from $5 \mathrm{~cm}$ to $55 \mathrm{~cm}$ with DC classes of $5 \mathrm{~cm}$. The equation developed can be part of growth systems and timber yield for the sustainable management of this species in the study area.

KEYWORDS: stem analysis, diameter at breast height, GADA equations, CAI-MAI, Korf model, time of passage. 


\section{INTRODUCCIÓN}

El crecimiento e incremento en las dimensiones de los árboles de especies maderables comerciales se predice mediante modelos de crecimiento teóricos, estos son la base científica que permite planificar estrategias de manejo silvícola y realizar aprovechamientos sostenibles en el tiempo. Por esta razón, es necesario desarrollar herramientas para generar información actualizada sobre la dinámica de crecimiento de las especies forestales de interés.

El diámetro normal es una de las variables más importantes de los árboles; se mide para tener conocimiento sobre su tamaño (Kjell y Lennart, 2005), es fácil de medir y tiene alta correlación con otros atributos del árbol y del rodal (Bueno-López y Bevilacqua, 2013), por ello es relevante su modelización para realizar estimaciones confiables y tomar decisiones informadas al planear y ejecutar las prácticas silvícolas.

Los modelos de crecimiento en diámetro de árboles individuales son uno de los componentes esenciales de sistemas de crecimiento y rendimiento maderable, que posibilitan planear el manejo y el aprovechamiento forestal al simular el desarrollo y producción de los rodales bajo diferentes alternativas silvícolas (Xu et al., 2014; Moreno, Palmas, Escobedo, Cropper y Gezan, 2017). Estos modelos permiten estimar el tiempo mínimo requerido para que los árboles alcancen un determinado diámetro normal y puedan ser aprovechados comercialmente bajo diferentes condiciones silvícolas (Quiñonez, De los Santos y Álvarez, 2015)

En México, estos modelos tienen utilidad en la planeación y ejecución de programas de manejo forestal maderable, ya que permiten determinar en forma objetiva las tasas de crecimiento en diámetro normal (incrementos), los tiempos de paso por categoría diamétrica y el turno técnico o comercial; además, marcan la pauta para determinar el ciclo de corta en función de los diámetros deseados para la cosecha final.

Para generar modelos de crecimiento en diámetro se puede utilizar información generada a partir de la técnica de análisis troncal, la cual ha sido una parte integral de la medición del bosque y ha tenido amplias aplicaciones en silvicultura por más de un siglo para modelar diferentes parámetros de crecimiento; además, proporciona series de patrones de crecimiento individuales; esta información cronológica se usa para estimar y predecir el crecimiento en diámetro a diferentes edades de los árboles (Pretzsch, Dauber y Biber, 2013).

Pinus montezumae Lamb. (pino real) es una conífera endémica de México, con amplia distribución y abundancia en el Eje Neovolcánico Transversal, en la región "IxtaPopo" ocupa el primer lugar en importancia para el aprovechamiento comercial maderable, domina en $70 \%$ de la superficie forestal bajo producción (102 968 ha formando rodales coetáneos uniespecíficos y mixtos junto a otros taxones de Pinus; su madera se usa para la construcción, producción de chapa, celulosa, papel, durmientes, postes, muebles y duelas (Comisión Nacional Forestal [Conafor], 2012). Los árboles alcanzan alturas de $25 \mathrm{~m}$ a $30 \mathrm{~m}$, crecen en una altitud promedio de $2500 \mathrm{~m}$, con una precipitación media anual de 800 mm (Perry, 1991). Aun con el escenario e importancia referidos, son limitados los estudios orientados a generar herramientas silvícolas cuantitativas que permitan modelar y estimar el crecimiento e incremento de esta especie.

\section{OBJETIVOS}

El objetivo de este trabajo fue desarrollar una ecuación dinámica que estime el crecimiento e incremento en diámetro normal para árboles individuales de Pinus montezumae Lamb. que conforma bosques coetáneos en la región "Ixta-Popo" de Puebla, México.

\section{MATERIALES Y MÉTODOS}

Este estudio se realizó en la Unidad de Manejo Forestal 2101, que corresponde a la región "Ixta-Popo" en Puebla, México, localizada entre $18.45^{\circ}$ y $19.50^{\circ} \mathrm{N}$ y $97.60^{\circ}$ y $98.75^{\circ}$ O. Se ubica en la provincia fisiográfica X Eje Neovolcánico, en la parte alta de la región hidrológica 18 Río Balsas. Las comunidades vegetales principales son bosques de pino, de oyamel, de pino-encino y de encino, se ubican de $1800 \mathrm{~m}$ a 
2900 m s.n.m. y se gestionan con sistemas de manejo regular e irregular. El clima predominante es templado, subhúmedo $\mathrm{C}(\mathrm{w} 2)$ con temperatura media anual entre 12 ${ }^{\circ} \mathrm{C}$ y $18{ }^{\circ} \mathrm{C}$, temperatura del mes más frío de $-3{ }^{\circ} \mathrm{C}$ y del más caliente de $22{ }^{\circ} \mathrm{C}$; lluvias de verano con índice $\mathrm{P} / \mathrm{T}$ mayor de 55 con precipitación en el mes más seco menor a $40 \mathrm{~mm}$. La pendiente media es de 30\% a 40\%. Los suelos de mayor importancia son de tipo regosol, cambisol y feozem (Servicio y Consultoría Ambiental y Forestal [SCAF], 2011).

Se utilizó información de diámetro normal sin corteza $(\mathrm{Dn}=\mathrm{Y} ; \mathrm{cm})$ y edad $(\mathrm{E}=\mathrm{t}$; años $)$ generada de una muestra de 81 árboles de Pinus montezumae colectada en predios forestales de la unidad de manejo referida. Los árboles seleccionados fueron dominantes y codominantes en altura, libres de defectos, sanos y de fuste recto; incluyeron diferentes categorías de diámetro normal y de altura total y cubrieron diferentes calidades de estación.

Cada árbol fue derribado y seccionado, se obtuvieron rodajas de $5 \mathrm{~cm}$ de espesor a la altura del tocón $(0.3 \mathrm{~m})$, a la del diámetro normal $(1.3 \mathrm{~m})$ y posteriormente a medidas comerciales de $2.5 \mathrm{~m}$ hasta la primera rama viva del árbol, después a cada metro hasta que el diámetro mínimo en el fuste fue de $5 \mathrm{~cm}$. Para medir los anillos anuales de crecimiento y determinar la edad a diferentes diámetros para cada árbol, se aplicó la técnica de análisis troncal (Klepac, 1976; Pretzsch et al., 2013). Con los pares de datos de diámetro normal y edad se conformó una base de datos que fue auditada en forma gráfica para verificar consistencia y comportamiento lógico de las variables.

En la tabla 1 se presenta un resumen de los estadísticos básicos de las variables analizadas en la muestra, compuesta por 81 series de crecimiento equivalentes a 648 pares de datos.

A los pares de datos se ajustaron, por regresión estadística, seis ecuaciones dinámicas $(\mathrm{EcD})$ que en un análisis previo resultaron plausibles (Tabla 2), las cuales fueron generadas al aplicar el enfoque de la diferencia algebraica generalizada (DAG) (Cieszewski y Bailey, 2000). Las primeras tres ecuaciones generan curvas polimórficas y son equivalentes al enfoque de diferencia algebraica (DA)
(Bailey y Clutter, 1974); para las últimas se combinan las hipótesis de que tanto la tasa de crecimiento como el potencial máximo de crecimiento varían entre sitios, lo que da lugar a curvas de crecimiento polimórficas con asíntotas variables.

Por convención en la notación, las ecuaciones dinámicas en DAG tienen la estructura general implícita de la forma: $Y=\mathrm{f}\left(t, t_{0}, Y_{0}, B_{1}, B_{2}, B_{m}\right)$, donde $Y$ es el diámetro normal promedio por estimar a la edad $t, B_{1}, B_{2}, B_{m}$ son los parámetros globales; $Y_{0}$ es el diámetro normal observado a la edad $t_{0}$; tanto $Y_{0}$ como $t_{0}$ quedan definidos como condiciones iniciales y $Y_{0}$ puede ser definido como parámetro durante el ajuste (Cieszewski y Bailey, 2000).

Las EcD1 y EcD2 usan como modelos base al derivado por Yang, Kozak y Smith (1978) y por McDill y Amateis (1992), respectivamente; las EcD3 y EcD4 toman como modelo base al generado por Chapman-Richards (Chapman, 1961; Richards, 1959); en tanto que la EcD5 usa el modelo base de Korf (1939) y la EcD6 corresponde a un caso especial de las clases de modelo tipo log-logístico y es equivalente al modelo de Hossfeld (Cieszewski, 2000).

En una primera etapa, el ajuste estadístico de las ecuaciones se realizó con la base de datos bajo un arreglo en pares no traslapados; se usó el método de máxima verosimilitud con información completa (FIML) con el procedimiento Model del paquete estadístico SAS/ETS v. 9.3 (SAS Institute Inc., 2011).

Para seleccionar la mejor EcD se realizó: (1) un análisis de la calidad de ajuste basado en comparaciones numéricas de estadísticos de bondad y, (2) una evaluación del realismo biológico mediante una comparación gráfica de las tendencias de las curvas que cada ecuación describió para la condición promedio y para los límites superior e inferior, sobrepuestas a las trayectorias de crecimiento observadas (Quiñonez et al., 2015). Se evaluaron los estadísticos de bondad de ajuste: coeficiente de determinación ajustado por el número de parámetros $\left(\mathrm{R}^{2}{ }_{\text {adj }}\right)$, la raíz del cuadrado medio del error (RCME), el sesgo, el logaritmo de la verosimilitud (logLik) y el criterio de información de Akaike (AIC), expresados en la forma siguiente: 


$$
\begin{gathered}
R_{a d j}^{2}=1-\left(1-R^{2}\right)\left(\frac{n-1}{n-p}\right) \operatorname{con}: R^{2}=1-\frac{\sum_{i=1}^{n}\left(Y_{i}-\hat{Y}_{i}\right)^{2}}{\sum_{i=1}^{n}\left(Y_{i}-\bar{Y}_{i}\right)^{2}} \\
R C M E=\sqrt{\frac{\sum_{i=1}^{n}\left(Y_{i}-\hat{Y}_{i}\right)^{2}}{n-p}} \\
\text { Sesgo }=\sum_{i=1}^{n} \frac{Y_{i}-\hat{Y}_{i}}{n} \\
\text { AIC }=2 p+n \ln \left(\frac{\sum_{i=1}^{n}\left(Y_{i}-\hat{Y}_{i}\right)^{2}}{n}\right)
\end{gathered}
$$

donde:

$\hat{Y}_{i}, Y_{i}$ y $\bar{Y}_{i}$ son los valores estimados, observados y promedios, respectivamente

$n$ es el número total de observaciones usadas en el ajuste

$p$ es el número de parámetros en cada EcD

Para apoyar la evaluación numérica de la calidad de ajuste, sobre los estadísticos se aplicó un sistema de calificación relativa, consistente en jerarquizar cada estadístico de las EcD conforme a Sakici, Misir, Yavuz y Misir (2008) para obtener una calificación total para cada ecuación. La mejor ecuación dinámica fue aquella que presentó un balance entre consideraciones estadísticas y razones biológicas.

En una segunda etapa, los parámetros de la ecuación dinámica seleccionada se estimaron con el método de variables artificiales (dummy variable method) propuesto por Cieszewski, Harrison y Martin (2000), el cual es invariante respecto a la edad de referencia, lo que implica que el ajuste no depende de la asignación previa de una edad de referencia y por tanto no se obliga al modelo a pasar por punto edad-diámetro alguno. Por el contrario, este método toma en cuenta los errores de medición y los errores aleatorios de las unidades experimentales; a partir de las tendencias individuales, estima parámetros específicos para cada árbol y parámetros globales, comunes a todos los individuos y que corresponden a los de la EcD; además, usa la información completa de las observaciones (Cieszewski, 2003; Diéguez-Aranda, Burkhart y Rodríguez-Soalleiro, 2005; Vargas-Larreta, Álvarez-González, Corral-Rivas y Aguirre, 2010).

Por la naturaleza de los datos observados que corresponden a múltiples mediciones por unidad experimental, con estructura jerárquica y longitudinal, se presentan los fenómenos de: (1) errores autocorrelacionados por dependencia espacial dentro de cada unidad experimental y (2) varianza no constante de los errores, lo que viola los supuestos básicos de la regresión. Para corregir estos problemas, la estructura de covarianza que implica al término del error $\left(e_{i j}\right)$ se modeló mediante una estructura continua autoregresiva AR(p) de orden $\mathrm{p}$ (Zimmerman et al., 2001). Bajo este supuesto, el término de error se expresó en la forma siguiente:

$$
e_{i j}=\sum_{k=1}^{k=x} I_{k} \rho_{k}^{t_{i j}-t_{i j-k}} e_{i j-k}+\varepsilon_{i j}
$$

donde:

$e_{i j}$ es el j-ésimo residuo ordinario del i-ésimo árbol

$e_{i j-k}$ es el j-ésimo residuo ordinario del i-k-ésimo árbol

$I_{k}=1$ para $\mathrm{j}>\mathrm{k}$ y es cero para $\mathrm{j} \leq \mathrm{k}$

$\rho_{k}$ es el parámetro autorregresivo de orden k a ser estimado $t_{i j}-t_{i j-k}$ es la distancia que separa la j-ésima de la j-késima observación dentro de cada árbol

$t_{i j}>t_{i j-k} \cdot \varepsilon_{i j}$ es el término del error bajo la condición de independencia

TABLA 1. Estadísticas descriptivas de las variables analizadas para la muestra analizada.

\begin{tabular}{lccccc}
\hline Variable & Media & Mínimo & Máximo & DE & CV \\
\hline Diámetro normal (Dn; cm) & 28.87 & 0.00 & 65.30 & 14.8413 & 51.4099 \\
Edad (E; años) & 40.00 & 10.00 & 70.00 & 20.0177 & 50.0442 \\
\hline
\end{tabular}

Donde DE: desviación estándar (m); CV: coeficiente de variación (\%). 
TABLA 2. Formulaciones de las ecuaciones dinámicas evaluadas para el ajuste de crecimiento en diámetro normal.

\begin{tabular}{|c|c|c|c|c|}
\hline Modelo base & $\begin{array}{l}\text { Parámetros } \\
\text { relacionados al } \\
\text { sitio }\end{array}$ & $\begin{array}{l}\text { Solución para } X \text { con valores iniciales } \\
\text { en to y } Y_{0}\end{array}$ & Ecuación dinámica & \\
\hline $\begin{array}{c}\text { Weibull } \\
Y=a\left(1-\exp \left(-b t^{c}\right)\right)\end{array}$ & $b=X$ & $X_{0}=-\frac{\ln \left(1-\left(Y_{0} / B_{1}\right)\right)}{t_{0}^{B_{3}}}$ & $Y=B_{1}\left(1-\left(1-Y_{0} / B_{1}\right)^{\left(t / t_{0}\right)^{B_{3}}}\right)$ & (EcDl) \\
\hline$Y=\frac{a}{1+\exp (b) t^{c}}$ & $b=X$ & $X_{0}=\frac{-B_{2} t_{0}^{-B_{3}}}{1-B_{1} Y_{0}^{-1}}$ & $Y=\frac{B_{1}}{1-\left(1-B_{1} Y_{0}^{-1}\right)\left(t_{0} / t\right)^{B_{3}}}$ & (EcD2) \\
\hline \multirow[t]{2}{*}{$\begin{array}{l}\text { Chapman-Richards } \\
Y=a\left(1-\exp (-b t)^{c}\right.\end{array}$} & $b=X$ & $X_{0}=\frac{-\ln \left(1-\sqrt[B_{3}]{Y_{0} / B_{1}}\right)}{t_{0}}$ & $Y=B_{1}\left(1-\left(1-\sqrt[B_{3}]{Y_{0} / B_{1}}\right)^{t / t_{0}}\right)^{B_{3}}$ & (EcD3) \\
\hline & $\begin{array}{c}a=\exp (X) \\
c=B_{2} / X\end{array}$ & $\begin{array}{l}L_{0}=\ln \left(1-\exp \left(-B_{2} t_{0}\right)\right) \\
R_{0}=\sqrt{\ln \left(Y_{0}\right)^{2}-4 B_{3} L_{0}} \\
X_{0}=0.5\left(\ln \left(Y_{0}\right)+R_{0}\right)\end{array}$ & $Y=\exp \left(X_{0}\right)\left(1-\exp \left(-B_{2} \mathrm{t}\right)\right)^{\left(B_{3} / \mathrm{X}_{0}\right.}$ & (EcD4) \\
\hline $\begin{array}{c}\text { Korf } \\
Y=\mathrm{a} \exp \left(-b t^{-c}\right)\end{array}$ & $\begin{array}{c}a=\exp (X) \\
b=B_{1}+B_{2} / X\end{array}$ & $\begin{array}{l}R_{0}=\sqrt{\left(B_{1} t_{0}^{-B_{3}}+\ln \left(Y_{0}\right)\right)^{2}+4 B_{2} t_{0}^{-B_{3}}} \\
X_{0}=0.5 B_{1} t_{0}^{-B_{3}}+\ln \left(Y_{0}\right)+R_{0}\end{array}$ & $Y=\exp \left(X_{0}\right) \exp \left(\left(-B_{1}+\frac{B_{2}}{X_{0}}\right) t^{-B_{3}}\right)$ & (EcD5) \\
\hline$Y=\frac{a}{1+b t^{-c}}$ & $\begin{array}{l}a=B_{1}+X \\
c=B_{2} X\end{array}$ & $X_{0}=\frac{Y_{0}-B_{1}}{1-B_{2} Y_{0} t^{-B_{3}}}$ & $Y=\frac{B_{1}+X_{0}}{1+B_{2} X_{0} t^{-B_{3}}}$ & (EcD6) \\
\hline
\end{tabular}

Donde $Y$ : es el diámetro normal a la edad $t ; Y_{0}$ : es el diámetro normal a la edad $t_{0}$; ln: es logaritmo natural; exp: es la función exponencial; a, b y c: son parámetros de cada modelo base; Bi: son parámetros de cada ecuación dinámica a ser estimados.

El problema de heterocedasticidad se solucionó al considerar que la varianza del error $\left(\sigma^{2}\right)$ puede modelarse como una función potencia del diámetro normal predicho $(\widehat{D n}$ $=$ pred.y) (Parresol, 1993), el factor de ponderación $\left(W_{i}\right)$ que se utilizó fue $W_{i}=\widehat{D n}_{1}^{k}$ para lograr la homogeneidad de la varianza, con actualización dinámica de los residuos (resid.y), $k$ tomó el valor constante de 0.5 , la estructura de la varianza que se usó en el ajuste se expresó en la forma:

$$
\text { resid. } y=\text { resid. } y\left(\text { pred. } y^{0.5}\right)^{0.5}
$$

La estimación de los parámetros de la $\mathrm{EcD}$, junto con la estructura de covarianza y varianza del error, se programó para un ajuste simultáneo con el procedimiento Model de SAS/ETS (SAS Institute Inc., 2011). La corrección de la autocorrelación se verificó con el estadístico de DurbinWatson (DW), el cual indica que no existe correlación espacial o temporal cuando el valor de DW es próximo a 2 (Draper y Smith, 1998).

La calidad de ajuste de la ecuación dinámica corregida por autocorrelación y heterocedasticidad se comparó contra el ajuste sin corregir, se usaron los estadísticos: coeficiente de determinación ajustado $\left(\mathrm{R}_{\text {adj }}^{2}\right.$, logaritmo de verosimilitud (logLik), comportamiento gráfico del sesgo y de la raíz del cuadrado medio del error (RCME) por clase diamétrica, así como una inspección visual de los residuales contra los predichos y la edad.

La EcD corregida se usó para construir un índice de diámetro normal (IDn) como medida de productividad, análogo al concepto de índice de sitio, esto es posible siempre que los árboles de la muestra procesada sean dominantes y codominantes en altura, tal como lo fue en el presente estudio. El IDn se definió como el diámetro 
normal promedio que alcanza la especie para una edad de referencia de 50 años. Con este índice se construyó una familia de curvas de crecimiento en Dn para clasificar la productividad en baja, media y alta. Con base en Prodan, Peters, Cox y Real (1997) y en la aplicación de cálculo diferencial, se derivaron las expresiones para determinar los incrementos corriente y medio anual (ICA e IMA) y sus respectivos valores máximos para cada IDn. Finalmente, se generó la expresión para determinar y analizar el tiempo de paso (TP), que corresponde al tiempo en años que le toma al árbol para pasar de una categoría diamétrica dada a la inmediata superior.

\section{RESULTADOS Y DISCUSIÓN}

Los parámetros en todas las ecuaciones dinámicas fueron significativamente diferentes de cero $(p<0.05)$ (Tabla 3); aunque todos los ajustes son aceptables, en esta primera instancia es prematuro seleccionar alguna $\mathrm{EcD}$ en particular para estimar el crecimiento en Dn.

Los estadísticos de bondad de ajuste de las ecuaciones evaluadas evidencian altos valores en el $\mathrm{R}^{2}$ adj $(>92 \%$ ), precisiones dadas por la RCME que fluctúan de $2.44 \mathrm{~cm} \mathrm{a}$ $3.43 \mathrm{~cm}$ y bajos sesgos que varían de $0.14 \mathrm{~cm}$ a $0.58 \mathrm{~cm}$. Los valores del $\operatorname{logLik}$ y del AIC fueron similares, excepto en las ecuaciones EcD1 y EcD6 que fueron menos aceptables (Tabla 4). Estos resultados, junto con la calificación total de cada ecuación, sugieren que comparati-vamente las ecuaciones EcD2, EcD3 y EcD4 podrían ser opciones para modelar con mayor precisión el crecimiento en diámetro normal.

Sin embargo, un comparativo mediante inspección visual sobre el comportamiento gráfico respecto a las tendencias que cada ecuación describe determinó que las ecuaciones EcD2, EcD5 y EcD6 se sobreponen de mejor manera a las tendencias de los datos observados, sobre todo en los extremos superior e inferior de los valores de Dn en todo el intervalo de edad analizado (Fig. 1). Las ecuaciones EcD1 y EcD4 generaron curvas de crecimiento con tendencia a subestimar en forma acentuada en el extremo superior de edad observado; en cambio, las curvas que generó la EcD3 tendieron a sobreestimar en ese mismo extremo de edad, separándose marcadamente de las tendencias observadas en los límites superior e inferior del diámetro normal.

Aun cuando la EcD5 ocupó el cuarto lugar en la calificación total, con valores en el $\mathrm{R}_{\text {adj }}^{2}$ y en la RCME muy cercanos a los de las mejores ecuaciones (Tabla 4), gráficamente mostró que tiene la mejor capacidad predictiva y un comportamiento biológicamente realista, puesto que las tendencias de las curvas que genera se sobreponen de mejor manera a todo lo largo y ancho de las trayectorias de crecimiento observadas, lo que evidencia que posee una lógica consistente y razonable (Fig. 1), aspectos por los que se seleccionó como la mejor EcD para estimar el diámetro normal sin corteza, así como para su posterior refinamiento en la técnica de ajuste.

La EcD5 también fue seleccionada por Gea-Izquierdo, Cañellas y Montero (2008), para modelar el crecimiento en diámetro normal de Quercus ilex en el centro-oeste de España, y por Barrio et al (2006), para generar un sistema de crecimiento en área basal para Pinus pinaster en el noroeste de España. Esta coincidencia en seleccionar la misma ecuación confirma que la variante en DAG del modelo de Korf (con $a=\exp (X)$ y $\left.b=B_{1}+B_{2} / X\right)$ es conveniente para describir con precisión el crecimiento en diámetro normal. Además, también ha demostrado ser eficiente para predecir el crecimiento en altura dominante y estimar el índice de sitio, ya que fue seleccionada por Vargas-Larreta, AguirreCalderón, Corral-Rivas, Crecente-Campo y Diéguez-Aranda (2013) e Hirigoyen, Franco y Diéguez (2018), para Pinus pseudostrobus. en el noreste de México y para Eucalyptus globulus en el sur y sureste de Uruguay, respectivamente.

La superioridad de la EcD5 se explica porque, al construirse con el enfoque de la DAG, los dos parámetros específicos de sitio la hacen más flexible para cubrir una amplia variedad de condiciones y tendencias de crecimiento al generar curvas polimórficas con asíntotas múltiples (Cieszewski y Bailey, 2000), lo que ratifica que este enfoque de modelado es matemáticamente robusto y biológicamente realista (Cieszewski, 2003; Sharma, Vacek, Vacek, Jansa y Kučera, 2017), características deseables para estimar el crecimiento de diversos atributos a nivel de árbol y de rodal. 
TABLA 3. Valores de los parámetros, errores estándar y nivel de significancia obtenidos en el ajuste de las ecuaciones dinámicas evaluadas.

\begin{tabular}{|c|c|c|c|c|c|}
\hline Ecuación dinámica & Parámetro & Estimador & Error estándar & Valor de $t$ & Significancia* \\
\hline EcD1 & $\mathrm{B}_{1}$ & 60.66843 & 3.0578 & 19.84 & $<0.0001$ \\
\hline \multirow[t]{2}{*}{ EcD2 } & $\mathrm{B}_{1}$ & 77.84524 & 3.3540 & 23.21 & $<0.0001$ \\
\hline & $\mathrm{B}_{3}$ & 1.430487 & 0.0295 & 48.42 & $<0.0001$ \\
\hline EcD3 & $\mathrm{B}_{3}$ & 1.456627 & 0.0366 & 39.84 & $<0.0001$ \\
\hline \multirow[t]{2}{*}{ EcD4 } & $\mathrm{B}_{2}$ & 0.023689 & 0.0016 & 14.54 & $<0.0001$ \\
\hline & $\mathrm{B}_{3}$ & 5.854212 & 0.1379 & 42.46 & $<0.0001$ \\
\hline EcD5 & $\mathrm{B}_{3}$ & 0.439907 & 0.0170 & 25.91 & $<0.0001$ \\
\hline \multirow[t]{3}{*}{ EcD6 } & $\mathrm{B}_{1}$ & 77.84452 & 6.5707 & 11.85 & $<0.0001$ \\
\hline & $\mathrm{B}_{2}$ & $3.58 \mathrm{E}+69$ & $2.06 \mathrm{E}-84$ & $1.74 \mathrm{E}+153$ & $<0.0001$ \\
\hline & $\mathrm{B}_{3}$ & 1.430495 & 0.0579 & 24.71 & $<0.0001$ \\
\hline
\end{tabular}

*Nivel de probabilidad 5\% ( $\alpha=0.05)$.

TABLA 4. Estadísticos de ajuste de las ecuaciones dinámicas evaluadas y calificación total.

\begin{tabular}{|c|c|c|c|c|c|c|}
\hline Ecuación dinámica & $R^{2} a d j$ & RCME & Sesgo & $\log L i k$ & $A / C$ & Calificación total \\
\hline EcD1 & 0.9212 & 3.4338 & 0.5559 & -1288 & 1201.13 & 29 \\
\hline EcD2 & 0.9573 & 2.4402 & 0.1725 & -1085 & 840.56 & 6 \\
\hline EcD3 & 0.9571 & 2.4485 & 0.1497 & -1087 & 843.75 & 9 \\
\hline EcD4 & 0.9539 & 2.5363 & 0.2142 & -1103 & 876.85 & 15 \\
\hline EcD5 & 0.9526 & 2.5718 & 0.2474 & -1109 & 890.92 & 20 \\
\hline EcD6 & 0.9218 & 3.4188 & 0.5808 & -1286 & 1197.87 & 26 \\
\hline
\end{tabular}




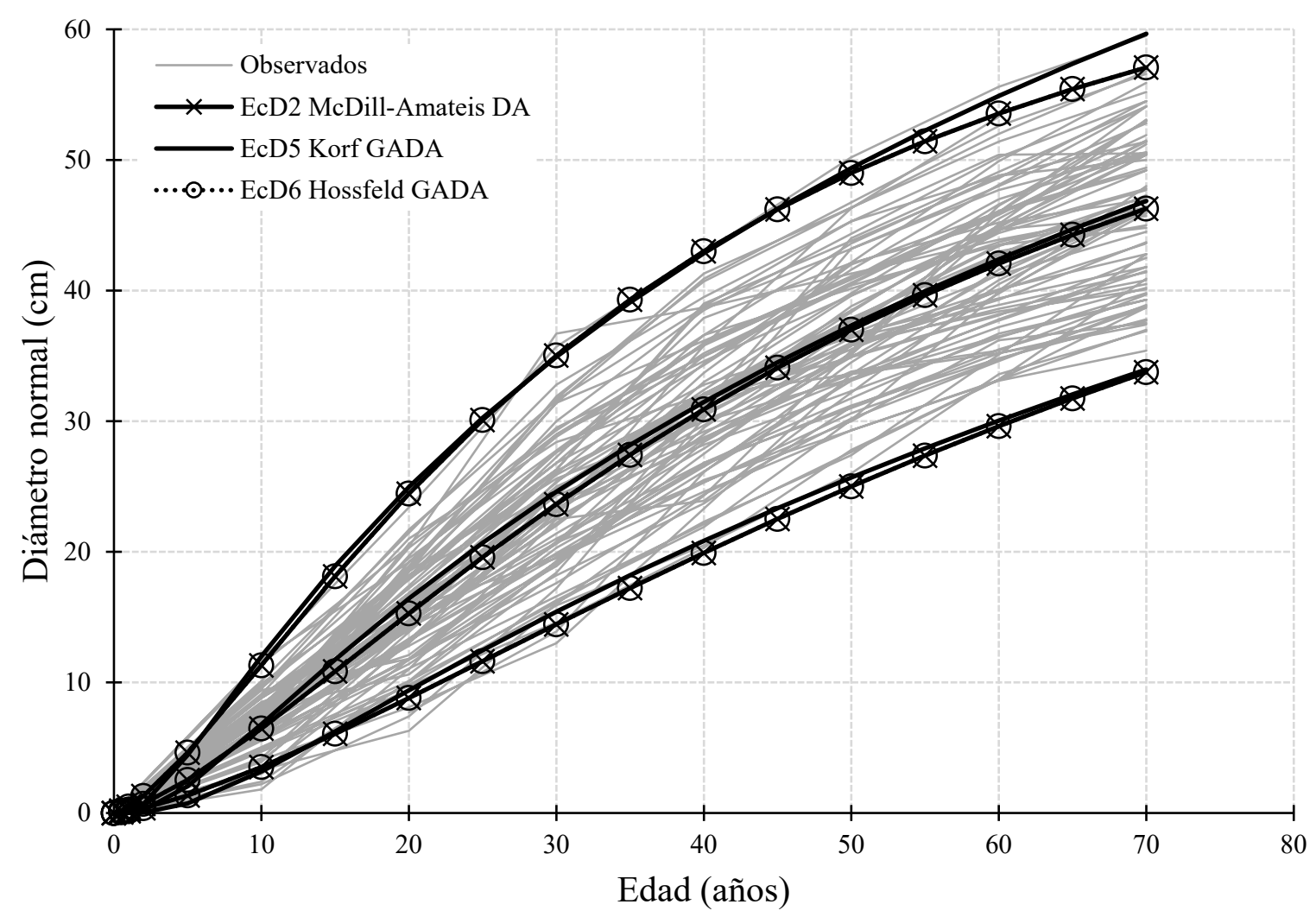

FIGURA 1. Comparativo de las tendencias que describen las mejores ecuaciones dinámicas para la condición promedio y límites superior e inferior con relación a las trayectorias observadas.

TABLA 5. Estadísticos de bondad ajuste de la EcD5 ajustada por el método de variables artificiales con corrección por autocorrelación y heterocedasticidad.

\begin{tabular}{crrrc}
\hline Parámetro & Estimador & Error estándar & Valor de t & Significancia \\
\hline $\mathrm{B}_{1}$ & $-3.69 \mathrm{E}+08$ & $4.05 \mathrm{E}+07$ & -9.12 & $<0.0001$ \\
$\mathrm{~B}_{2}$ & $1.82 \mathrm{E}+09$ & $3.42 \mathrm{E}+07$ & 53.28 & $<0.0001$ \\
$\mathrm{~B}_{3}$ & 0.590315 & 0.0361 & 16.34 & $<0.0001$ \\
$\rho_{1}$ & 0.318328 & 0.0171 & 18.59 & $<0.0001$ \\
$\rho_{2}$ & -0.415920 & 0.0242 & -17.19 & $<0.0001$ \\
\hline
\end{tabular}

Cuando la EcD5 se ajustó con el método de variables artificiales y se corrigió la autocorrelación con una estructura continua autoregresiva de orden $\operatorname{AR}(2)$ y la heterocedasticidad mediante la modelación de la varianza del error $\left(\sigma^{2}\right)$ como una función potencia de los predichos $(\widehat{D n})$, tanto los parámetros específicos como los globales, además del parámetro de autocorrelación ( $\varrho_{\mathrm{i}}$ ), fueron altamente significativos $(\alpha=0.05)$ (Tabla 5); los valores de $\mathrm{R}^{2}{ }_{\text {adj, }}$ RCME, sesgo, logLik y AIC fueron $0.9740,2.3930 \mathrm{~cm}$, $0.0154 \mathrm{~cm}, 1208$ y 852.09, respectivamente. El efecto de combinar el método de ajuste con ambas correcciones fue efectivo y favorable, permitió obtener estadísticos de ajuste superiores con respecto al ajuste efectuado por FIML y sin realizar las correcciones referidas (Tabla 4). 
La mejoría y ganancias en la calidad del ajuste también se pueden apreciar en forma gráfica al comparar los residuales contra los predichos que cada técnica de ajuste arroja; la corrección por heterocedasticidad presentó residuales que se distribuyen aleatoriamente en una franja comparativamente más estrecha alrededor de cero (Fig. 2a, b). Un comportamiento similar se obtuvo con la corrección de la autocorrelación, donde los residuales frente a la edad presentaron un patrón de distribución sin tendencia sistemática y en una escala menor (Fig. 2c, d), esta mejoría se refuerza con el valor del estadístico de DW, el cual fue de 1.4962 antes de la corrección y de 1.9075 posterior a esta; este último es próximo a 2 , lo que evidencia que el fenómeno de autocorrelación se eliminó (Draper y Smith, 1998). Ambas correcciones hacen que los errores sean idénticos e independientemente distribuidos y con varianza constante, lo que mejora las propiedades estadísticas y predictivas de la ecuación dinámica, puesto que ahora los parámetros estimados son de varianza mínima, insesgados y más eficientes. Bajo el estatus de errores idénticos e independientemente distribuidos, se pueden determinar intervalos de confianza realistas y realizar en forma correcta los habituales contrastes estadísticos de significación sobre los parámetros (West, Ratkowsky y Davis, 1984; Kozak, 1997).

Adicionalmente, el comportamiento gráfico de la RCME y el sesgo para las estimaciones del diámetro normal por intervalos de clase diamétrica de $5 \mathrm{~cm}$ evidencian que tanto la precisión como la exactitud de la EcD5, obtenidas del ajuste por el método de variables artificiales y corregido por autocorrelación y heterocedasticidad, son comparativamente superiores a los obtenidos por FIML y sin corregir; la tendencia de los valores de ambos estadísticos para el primer caso tiene menor variabilidad y estos se distribuyen más próximos a la línea del cero (Fig. 2e, f).

El método de variables artificiales también ha sido usado con resultados eficientes por Gea-Izquierdo et al (2008) para ecuaciones de diámetro normal, por Barrio et al. (2006) para modelar el área basal y por Diéguez-Aranda et al. (2005), Vargas-Larreta et al (2010), Sghaier et al (2012) e
Hirigoyen et al (2018) para ajustar modelos dinámicos de altura dominante. En este sentido, Wang, Borders y Zhao (2008) determinaron que este método proporciona una calidad de ajuste equivalente a la que ofrece la técnica de modelos de efectos mixtos (MEM). De acuerdo con Cieszewski (2003), el método de variables artificiales corresponde a una versión simplificada de un MEM, ya que tanto la estructura matemática como el algoritmo de ajuste son menos complejos, además de que los parámetros locales (específicos) se agrupan en un solo nivel (árbol o parcela), condición en la que se obtiene la mejor calidad de ajuste (Xu et al., 2014).

La familia de curvas de crecimiento construidas con la EcD5, con una edad de referencia de 50 años para tres clases de productividad definidas por el IDn, demuestra que las clases de $29 \mathrm{~cm}, 37 \mathrm{~cm}$ y $45 \mathrm{~cm}$, elegidas y etiquetadas para clasificar la productividad del sitio como bajo, medio y alto, respectivamente, son apropiadas y describen en forma satisfactoria las trayectorias de crecimiento en diámetro normal observadas (Fig. 3). Esto significa que la EcD5 explica adecuadamente la variación del crecimiento de esta variable de acuerdo con las diferentes calidades de sitio, ya que esa variación la absorbe la variable independiente no observable $\mathrm{X}$, la cual contiene en forma implícita la productividad, dada por factores ambientales diversos (Cieszewski y Bailey 2000).

Las curvas de crecimiento construidas con el IDn mantienen consistencia lógica y comportamiento similar con las generadas bajo este mismo principio y también con ecuaciones en DAG para Quercus ilex por Gea-Izquierdo et al. (2008), para Q. suber por Sánchez-González, Cardillo, Camacho, Garriga y Cañellas (2009), ambas especies de España; así como para cinco especies del género Pinus del norte de México desarrolladas por De Almeida et al. (2019). La EcD5 también puede utilizarse bajo la perspectiva indicada por Quiñonez et al (2015), quienes, para seis especies de Pinus de México, los valores de IDn que aplicó correspondieron a diferentes categorías de diámetro normal con intervalos de $5 \mathrm{~cm}$. 


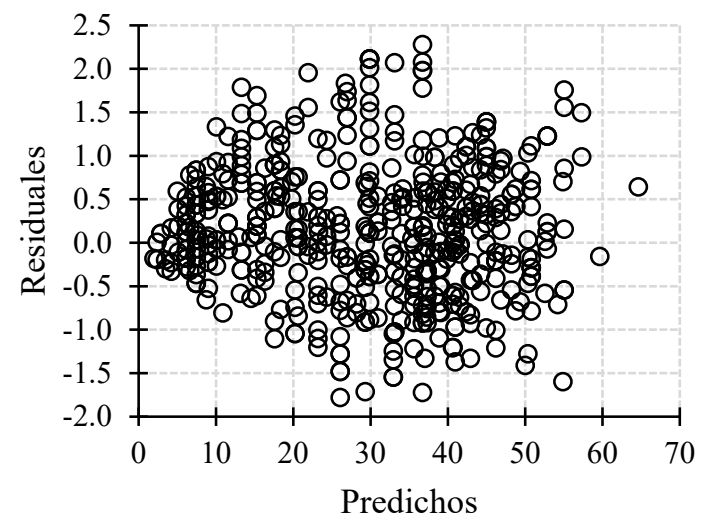

$\mathbf{a}$

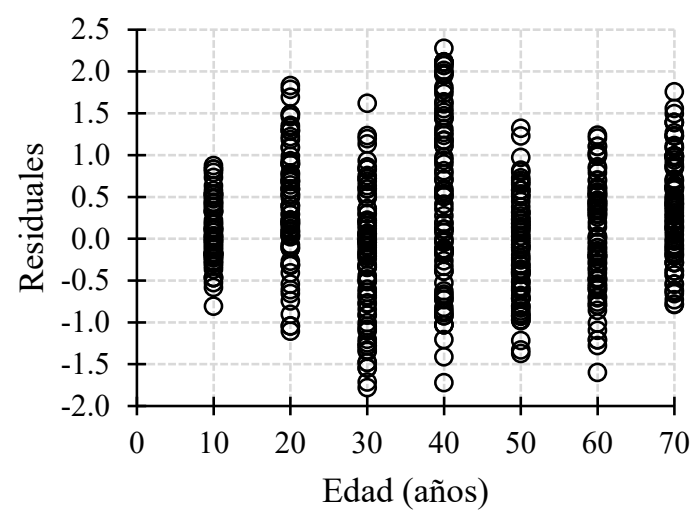

c

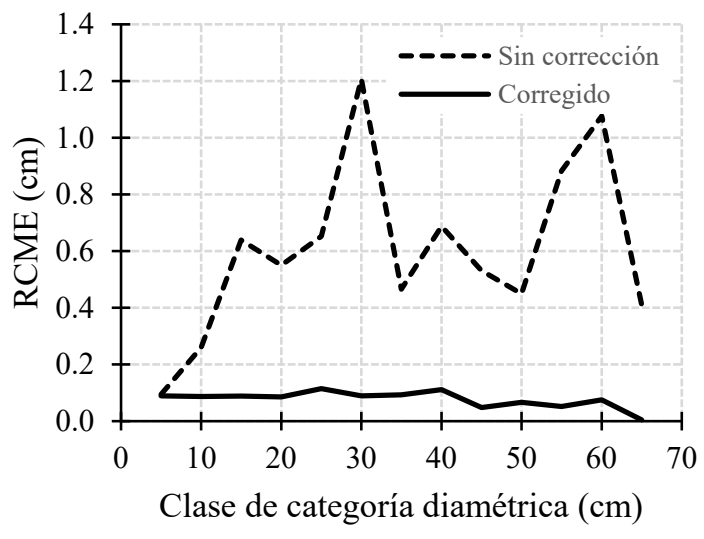

e

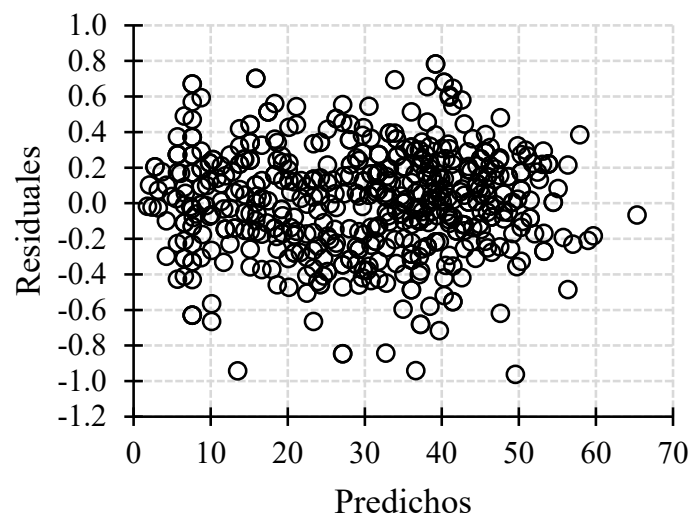

b

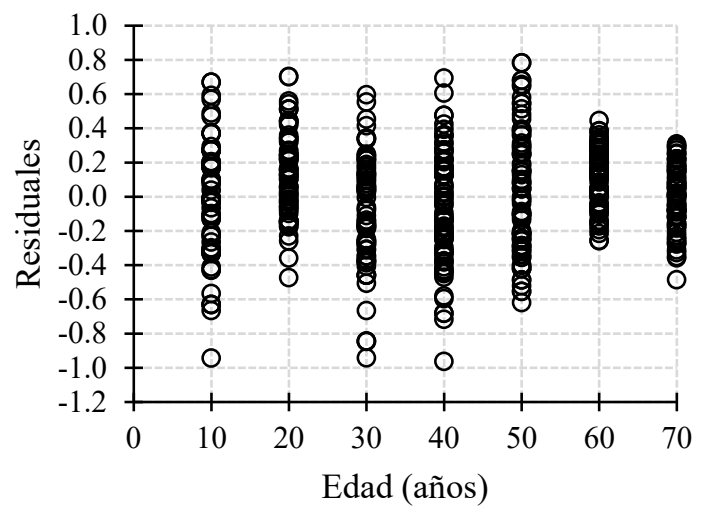

d

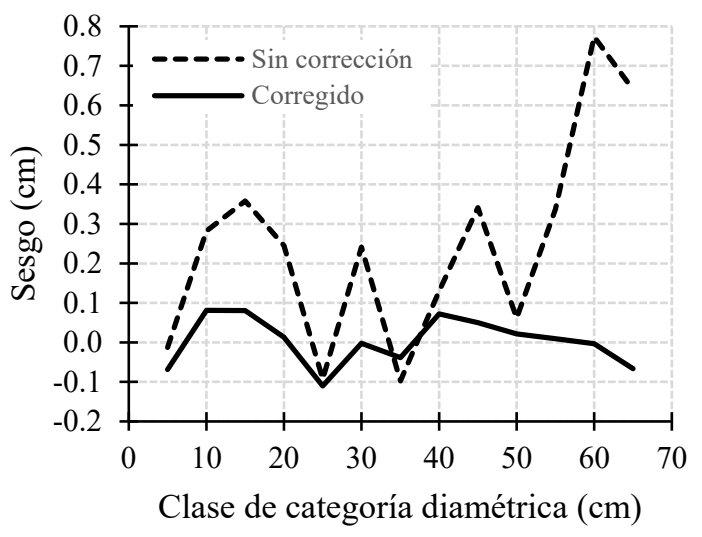

$\mathbf{f}$

Figura 2. Contraste de los residuales contra los predichos para la EcD5 con y sin corrección para la heterocedasticidad $(a, b)$, para la autocorrelación (c y d) y, ganancia en términos de la RCME y del sesgo por categoría diamétrica (e y f, respectivamente). 


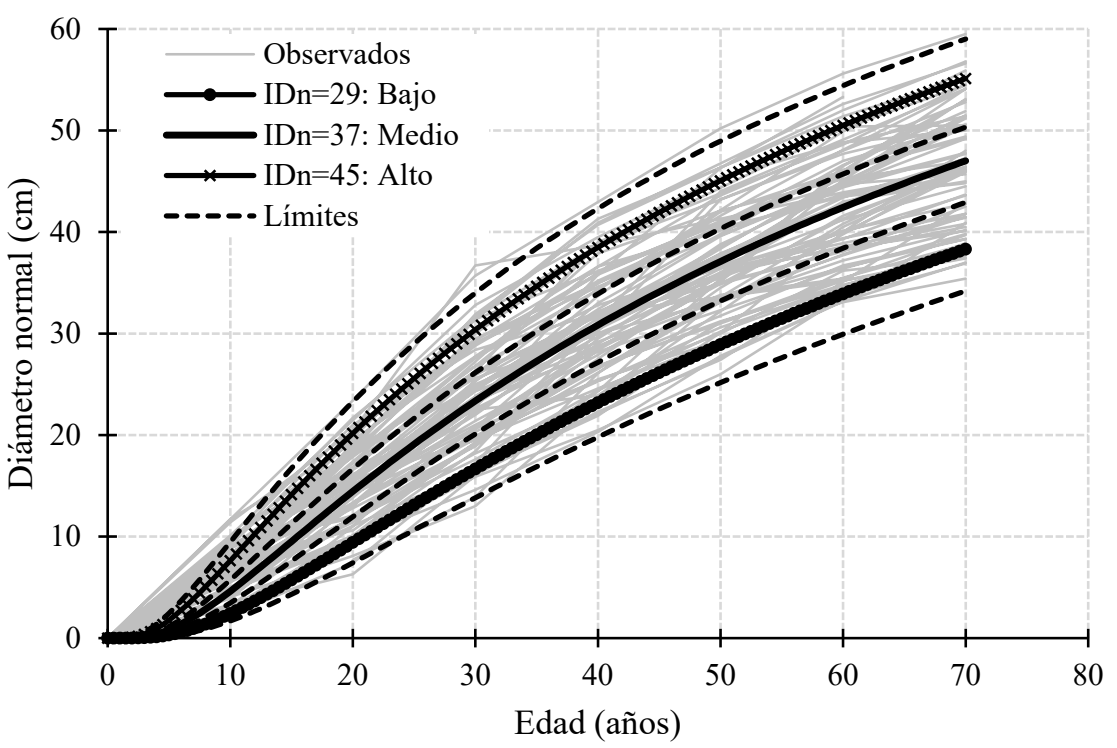

FIGURA 3. Curvas de crecimiento construida con la EcD5 y una edad de referencia de 50 años para tres niveles de productividad del sitio basadas en el índice de diámetro normal.

Las expresiones 7, 8, 9 y 10, cuyos componentes $X_{0}, R_{0}$ y $L_{0}$ se indican en la tabla 2, fueron derivadas de la EcD5 para estimar y construir curvas de incremento corriente anual (ICA), de ICA máximo ( $\mathrm{ICA}_{\text {máx }}$, de incremento medio anual (IMA) y de IMA máximo (IMA máx), respectivamente (Fig. 4). Con base en estas, se determinó que el $\mathrm{ICA}_{\operatorname{máx}}$ en Dn para árboles de $P$. montęumae es de $0.75 \mathrm{~cm}^{2} \tilde{n ̃ o}^{-1}, 1.00$ $\mathrm{cm}$ año-1 y $1.31 \mathrm{~cm}^{-1} \mathrm{n}^{-1}$ para los IDn bajo, medio y alto, respectivamente, en todos los casos corresponde a un Dn de $9.33 \mathrm{~cm}$, mismo que se alcanza a los 19.9 años, 14.8 años y 11.3 años, respectivamente. En tanto, el $\mathrm{IMA}_{\text {máx }}$ es de 0.58 $\mathrm{cm}, 0.78 \mathrm{~cm}$ y $1.02 \mathrm{~cm}$ para los IDn referidos, que corresponden a $25.37 \mathrm{~cm}$ de Dn, el cual se alcanza a los 43.64 años, 32.51 años y 24.79 años, respectivamente.

$$
\begin{gathered}
I C A=\frac{\exp \left(X_{0}\right)\left(B_{1}+\frac{B_{2}}{X_{0}}\right) t^{-B_{3}} B_{3} \exp -\left(\left(B_{1}+\frac{B_{2}}{X_{0}}\right) t^{-B_{3}}\right)}{t} \\
I C A_{\text {máx }}=\exp \left(-\frac{\ln \left(\frac{X_{0}\left(B_{3}+1\right)}{B_{3}\left(B_{1} X_{0}+B_{1}\right)}\right)}{B_{3}}\right) \\
I M A=\frac{\exp \left(X_{0}\right) \exp \left(\left(-B_{1}+\frac{B_{2}}{X_{0}}\right) t^{-B_{3}}\right)}{t}
\end{gathered}
$$

$$
I M A_{\text {máx }}=\exp \left(-\frac{\ln \left(\frac{X_{0}}{B_{3}\left(B_{1} X_{0}+B_{2}\right)}\right)}{B_{3}}\right)
$$

La tasa de crecimiento para la condición promedio es ligeramente superior a la obtenida por Hernández (2012) para esta misma especie en el sureste de Hidalgo, México; mediante el método de la curva guía determinó con el modelo de Korf que el ICA $_{\text {máx }}$ fue de $0.93 \mathrm{~cm}_{\text {año- }}{ }^{-1} \mathrm{y}$ se alcanza a los 21 años; el $\mathrm{IMA}_{\text {máx }}$ fue de $0.65 \mathrm{~cm}_{\text {año-1 }}{ }^{-1}$ que se logra a los 41 años. Sin embargo, los incrementos en este estudio son menores a los que se estiman mediante la curva guía con el modelo de Chapman-Richards seleccionado por Pacheco, Santiago, Martínez y Ortiz (2016) para el mismo taxón en la región de Sola de Vega, Oaxaca, México; en donde el $\mathrm{ICA}_{\operatorname{máx}}$ fue de $1.1 \mathrm{~cm}$ año-1 y sucede a los 13.5

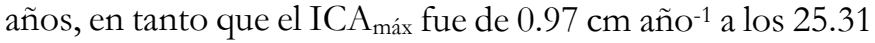
años. Las diferencias en estos atributos ratifican la necesidad de realizar estos estudios por especie y ecoregión para tener predicciones más confiables y así tomar decisiones de manejo silvícolas y de aprovechamiento maderable mejor fundamentadas. La ecuación desarrollada tiene aplicación práctica para rodales coetáneos de $P$. montęumae cuando el valor del índice de densidad del rodal máximo de Reineke sea de 0.31 a 0.73 . 


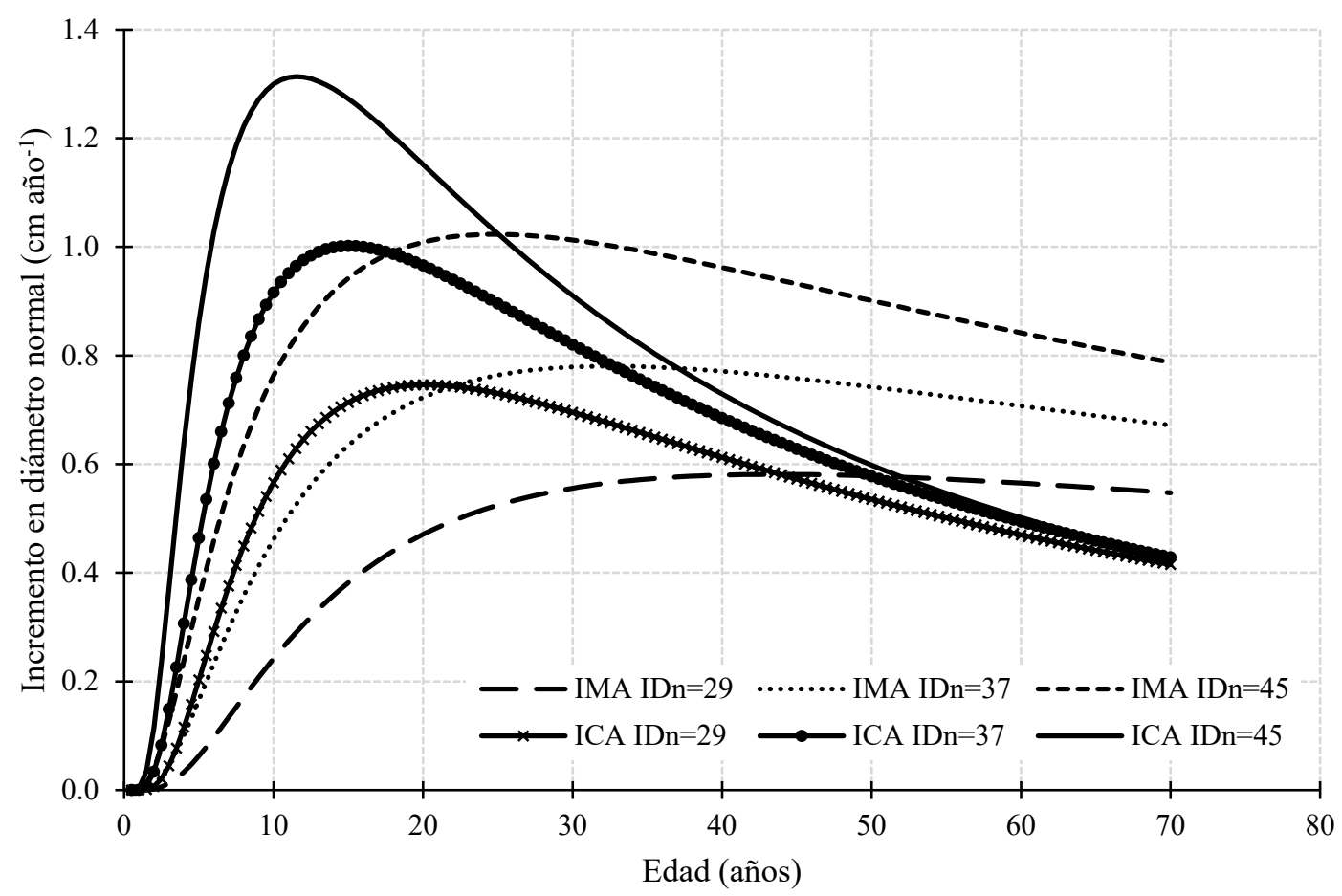

FIguRA 4. Comportamiento del ICA e IMA en diámetro normal por IDn obtenidos con la EcD5 para P. montezumae.

El ICA $A_{\text {máx }}$ en diámetro es un indicador de competencia y se considera como el momento adecuado para realizar los aclareos (Raymond, Joe, Anderson y Watt, 2008), ya que es mayor el impacto favorable a largo plazo en el crecimiento en diámetro normal (Kim et al., 2015); pues al reducirse la competencia, el incremento en diámetro aumenta debido a la mayor disponibilidad de recursos (luz, humedad, nutrientes y espacio) para el arbolado remanente. Por lo anterior, los primeros aclareos para rodales de $P$. montezumae en la zona de estudio deben iniciarse, en promedio, entre los 11 años y los 20 años, según el IDn; con lo que se contribuirá a un manejo más efectivo para favorecer árboles de alta calidad maderable que contengan mayor volumen y un valor comercial más alto.

La EcD5 puede invertirse, de modo que, para cualquier IDn, pueda determinarse con la expresión 11 la edad $(t)$ a la que se logra obtener la dimensión de determinado Dn de interés comercial requerido por la industria (aserrío, chapa, etc.), lo que da lugar al concepto de turno tecnológico, en donde el tamaño del diámetro normal toma relevancia. Esta situación, en la praxis implica estimar los tiempos de paso (TP) por categoría diamétrica (CD).

$$
t=\left(\frac{\ln (\mathrm{Y})-X_{0}}{-B_{1}-\frac{B_{2}}{X_{0}}}\right)^{-1 / B_{3}}
$$

En este sentido, para los tres niveles de productividad basados en el IDn se determinaron los TP por categoría diamétrica de $5 \mathrm{~cm}$ en un intervalo de $5 \mathrm{~cm}$ a $55 \mathrm{~cm}$ (Tabla 6). Para la condición de crecimiento promedio que equivale al IDn $=37$, el TP medio de 8.09 años es menor comparado con los TP promedio indicados por Quiñonez et al. (2015) en el mismo intervalo de CD, los cuales son de 13.55 años, 15.77 años, 16.01 años, 17.67, 20.41 años y 12.24 años para P. arizonica, P. duranguensis, $P$. teocote, P. leiophylla, P. lumboltrii y $P$. ayacabuite, respectivamente; todas estas especies crecen en Durango, México; este escenario evidencia que 
comparativamente $P$. montezumae en Puebla tiene mayores tasas de crecimiento.

El comportamiento gráfico del tiempo acumulado de los TP por IDn para P. montezumae sugiere que la tendencia es no lineal (Fig. 5a); este aspecto es importante a tomar en cuenta al momento de realizar extrapolaciones en el TP de categorías diamétricas inferiores a superiores, ya que generalmente se adopta el supuesto de que el crecimiento del Dn es lineal entre categorías consecutivas, situación que pueden provocar un sesgo importante en la determinación del TP.

La tendencia no lineal del TP es acentuada en especies de coníferas, como puede constatarse con estudios realizados por Bravo-Iglesias et al. (2010) para P. cubensis, por Quiñonez et al. (2015) para P. duranguensis y por Sharma et al. (2017) para Picea abies (Fig. 5b).

TABLA 6. Tiempos de paso por categoría diamétrica e IDn para P. montezumae.

\begin{tabular}{ccccccc}
\hline$C D$ & $L I$ & CENTRO & $L S$ & $T P I D n=29$ & $T P I D n=37$ & $T P I D n=45$ \\
\hline 5 & 5 & 7.5 & 9.99 & 3.45 & 2.57 & 1.96 \\
10 & 10 & 12.5 & 14.99 & 6.73 & 5.01 & 3.82 \\
15 & 15 & 17.5 & 19.99 & 7.04 & 5.24 & 4.00 \\
20 & 20 & 22.5 & 24.99 & 7.67 & 5.71 & 4.36 \\
25 & 25 & 27.5 & 29.99 & 8.54 & 6.36 & 4.85 \\
30 & 30 & 32.5 & 34.99 & 9.64 & 7.18 & 5.48 \\
35 & 35 & 37.5 & 39.99 & 11.01 & 8.20 & 6.26 \\
40 & 40 & 42.5 & 44.99 & 12.70 & 9.46 & 7.21 \\
45 & 45 & 47.5 & 49.99 & 14.78 & 11.01 & 8.39 \\
50 & 50 & 52.5 & 54.99 & 17.35 & 12.93 & 9.86 \\
55 & 55 & 57.5 & 59.99 & 20.58 & 15.33 & 11.69
\end{tabular}

Donde CD: categoría diamétrica; LI y LS: límite inferior y superior de la CD; TP: tiempo de paso; IDn: índice de diámetro normal.

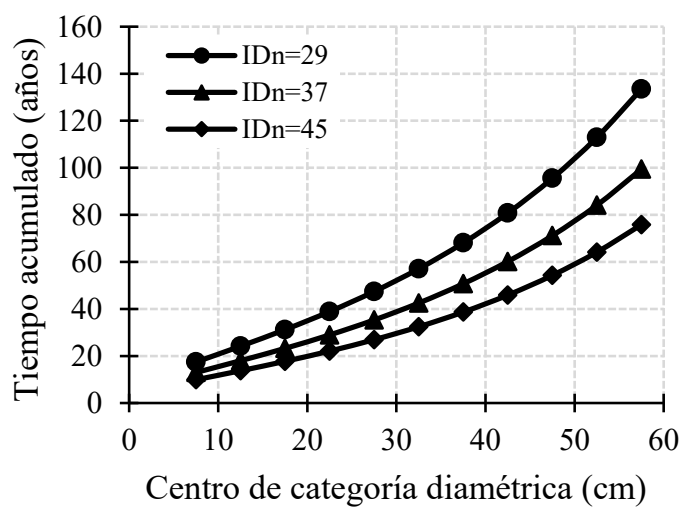

a

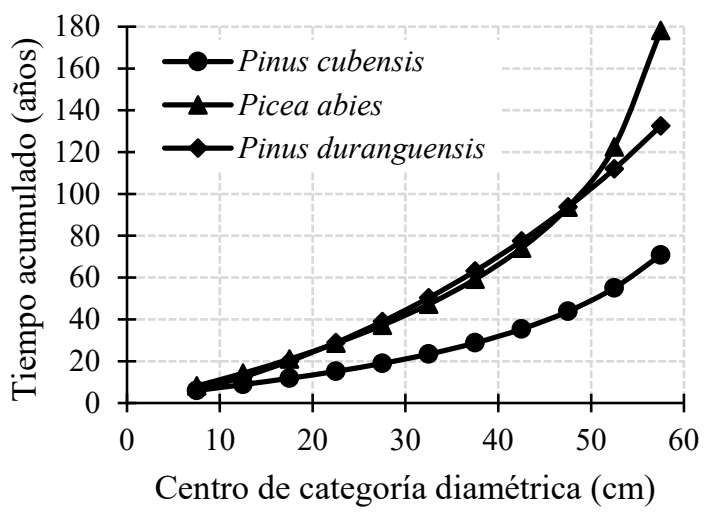

b

FIGURA 5. Tendencia del tiempo acumulado para que ocurra el cambio de categoría diamétrica por IDn y CD para P. montezumae (a) y contraste de la tendencia con respecto a otras especies de coníferas (b). 


\section{CONCLUSIONES}

Se desarrolló una ecuación dinámica expresada en diferencia algébrica generalizada para describir el crecimiento en diámetro de $P$. montęumae, ajustada mediante el método de variables artificiales y corregida por autocorrelación y heterocedasticidad. La ecuación posee alta calidad predictiva, es invariante de ruta y se basa en el modelo teórico de crecimiento de Korf. Es capaz de generar en forma simultánea curvas de crecimiento en diámetro polimórficas con asíntotas variables, lo que la hace altamente flexible para modelar una amplia variedad de condiciones de crecimiento, con predicciones biológicamente realistas.

Para tres niveles de productividad al aplicar el índice de diámetro normal (IDn $=29 \mathrm{~cm}, 37 \mathrm{~cm} \mathrm{y} 45 \mathrm{~cm}$, respectivamente), se determinó que el ICA $\mathrm{ICáx}_{\text {es }}$ de $0.75 \mathrm{~cm}$ año ${ }^{-1}, 1.00 \mathrm{~cm}_{\text {año }}{ }^{-1}$ y $1.31 \mathrm{~cm}^{2}$ año ${ }^{-1}$, que corresponden a un Dn de 9.33 cm, mismo que se alcanza a los 19.9 años, 14.8 años y 11.3 años, respectivamente. El $\mathrm{IMA}_{\operatorname{máx}}$ es de 0.58 $\mathrm{cm}, 0.78 \mathrm{~cm}$ y $1.02 \mathrm{~cm}$ para los IDn referidos, que corresponden a $25.37 \mathrm{~cm}$ de Dn, el cual se alcanza a los 43.64 años, 32.51 años y 24.79 años, respectivamente. Para el IDn $=37 \mathrm{~cm}$, que representa la condición de crecimiento promedio, el tiempo de paso medio se estimó en 8 años para un intervalo $5 \mathrm{~cm}$ a $55 \mathrm{~cm}$, con categorías diamétricas de $5 \mathrm{~cm}$. Por el conocimiento que esta ecuación genera, se puede considerar como una importante herramienta cuantitativa con base científica para planificar y mejorar la gestión forestal de rodales con esta especie.

\section{REFERENCIAS}

Bailey, R. L., \& Clutter, J. L. (1974). Base-age invariant polymorphic site curves. Forest Science, 20(2), 155-159. doi: 10.1093 /forestscience/20.2.155

Barrio A., M., Castedo D., F., Diéguez-Aranda, U., Álvarez G., J. G., Parresol, B. R., \& Rodríguez S., R. (2006). Development of a basal area growth system for maritime pine in northwestern Spain using the generalized algebraic difference approach. Canadian Journal of Forest Research, 36(6), 1461-1474. doi: 10.1139/X06-028

Bravo-Iglesias, J. A., Torres-Cárdenas, V., Rodríguez-Shade, L., ToiracArgüelles, W., Montalvo-Guerrero, J. M., Fuentes-Utría, V. M., \& Rodríguez-Cuevas, P. (2010). Modelación matemática del crecimiento del diámetro medio de Pinus cubensis Griseb en la empresa forestal integral Baracoa (parte I). Revista Forestal Baracoa, 29(1), 23-29.

Bueno-López, S., \& Bevilacqua, E. (2013). Diameter growth prediction for individual Pinus occidentalis Sw. trees. iForest, 6(4), 209-216. doi: 10.3832/ifor0843-006

Cieszewski, C. J. (2000). Analytical site index solution for the generalized log-logistic height equation. Forest Science, 46(2), 291-296. doi: 10.1093/forestscience/46.2.291

Cieszewski, C. J., Harrison, M., \& Martin, S. W. (2000). Practical methods for estimating non-biased parameters in self-referencing growth and yield models. University of Georgia PMRC-TR 2000-7. Athens, Georgia, USA. 12 p. Recuperado de http://www.pmrc.uga.edu/TR20007.pdf

Cieszewski, C. J., \& Bailey, R. L. (2000). Generalized algebraic difference approach: theory based derivation of dynamic equations with polymorphism and variable asymptotes. Forest Science, 46(1), 116126. doi: 10.1093 /forestscience/46.1.116

Cieszewski, C. J. (2003). Developing a well-behaved dynamic site equation using a modified Hossfeld IV function $\mathrm{Y}^{3}=\left(\mathrm{ax}^{\mathrm{m}}\right) /\left(\mathrm{c}+\mathrm{x}^{\mathrm{m}-}\right.$ 1), a simplified mixed-model and scant subalpine fir data. Forest Science, 49(4), 539-554. doi: 10.1093/forestscience/49.4.539

Comisión Nacional Forestal [Conafor]. (2012). Pinus montezumae Lamb. Recuperado de: http://www.conafor.gob.mx:8080/documentos/docs/13/971Pi nus $\% 20$ montezumae.pdf

Chapman, D. G. (1961). Statistical problems in population dynamics. In Neyman, J. (ed.) Proceedings of fourth Berkeley symposium on mathematical statistics and probability (pp. 153-168). Berkeley, USA: University of California Press.

De Almeida, S. M., Hernández V., M. R., Brazão, P. J. M., Escobar F., J. G., López S., C. A., Hernández D., J. C., \& Wehenkel, C. (2019). Sistema dinámico de crecimiento diamétrico para cinco especies de pinos en Durango, México. Revista Mexicana de Agroecosistemas, 6(2), 145-156.

Diéguez-Aranda, U., Burkhart, H. E., \& Rodríguez-Soalleiro, R. (2005). Modeling dominant height growth of radiata pine (Pinus radiata D. Don) plantations in north-western Spain. Forest Ecology and Management, 215, (1-3), 271-284. doi: 10.1016/j.foreco.2005.05.015

Draper, R. N., \& Smith, H. (1998). Applied regression analysis. New York, USA: John Wiley \& Sons, Inc.

Gea-Izquierdo, G., Cañellas, I., \& Montero, G. (2008). Site index in agroforestry systems: age-dependent and age-independent dynamic diameter growth models for Quercus ilex in Iberian open 
oak woodlands. Canadian Journal of Forest Research, 38(1), 101-113. doi: $10.1139 / \mathrm{X} 07-142$

Hernández, R. A. (2012). Determinación de turno e indice de sitio para Pinus montezumae Lamb., en el sureste del Estado de Hidalgo. Tesis de licenciatura, Universidad Autónoma Agraria Antonio Narro, Coahuila, México.

Hirigoyen, A., Franco, J., \& Diéguez, U. (2018). Modelo dinámico de rodal para Eucalyptus globulus (L.) en Uruguay. Agrociencia Uruguay, 22(1), 60-80. doi: 10.31285/agro.22.1.7

Kim, M., Lee, W. K., Kim, Y. S., Lim, C. H., Song, C., Park, T., Son, Y., \& Son, Y. M. (2015). Impact of thinning intensity on the diameter and height growth of Larix kaempferi stands in central Korea. Forest Science and Technology, 12(2), 77-87. doi: 10.1080/21580103.2015.1075435

Kjell, K., \& Lennart, N. (2005). Predicting the future diameter of stems in Norway spruce stands subjected to different thinning regimes. Canadian Journal of Forest Research, 35(6), 1331-1341. doi: $10.1139 / \mathrm{x} 05-058$

Klepac, D. (1976). Crecimiento e incremento de árboles y masas forestales. Chapingo, México: Universidad Autónoma Chapingo.

Korf, V. (1939). A mathematical definition of stand volume growth law. Lesnicka Prace 18, 337-379.

Kozak, A. (1997). Effects of multicollinearity and autocorrelation on the variables exponent taper functions. Canadian Journal of Forest Research, 27(5), 619-629. doi: 10.1139/x97-011

McDill, M. E., \& Amateis, R. L. (1992). Measuring forest site quality using the parameters of a dimensionally compatible height growth function. Forest Science, 38(2), 409-429. doi: 10.1093/forestscience/38.2.409

Moreno, P. C., Palmas, S., Escobedo, F. J., Cropper, W. P., \& Gezan, S. A. (2017). Individual-tree diameter growth models for mixed Nothofagus second growth forests in southern Chile. Forests, 8(506), 19. doi: $10.3390 /$ f8120506

Pacheco A., G., Santiago J., W., Martínez S., D., \& Ortiz B., R. (2016). Análisis del crecimiento e incremento y estimación de índice de sitio para Pinus montezumae Lamb. en Santiago Textitlán, Sola de Vega, Oaxaca. Foresta Veracruzana, 18(2), 21-28.

Parresol, B. R. (1993). Modeling multiplicative error variance: An example predicting tree diameter from stump dimensions in baldcypress. Forest Science, 39(4), 670-679.

Perry, J. P. Jr. (1991). The pines of Mexico and Central America. Portland, USA: Timber Press.

Pretzsch, H., Dauber, E., \& Biber, P. (2013). Species-specific and ontogeny-related stem allometry of european forest trees: evidence from extensive stem analyses. Forest Science, 59(3), 290302. doi: 10.5849 / forsci.11-102

Prodan, M., Peters, R., Cox, F., \& Real, P. (1997). Mensura Forestal. San José, Costa Rica: IICA y GTZ.

Quiñonez B., G., De los Santos P., H. M., \& Álvarez G., J. G. (2015). Crecimiento en diámetro normal para Pinus en Durango. Revista Mexicana de Ciencias Forestales, 6(29), 108-125. doi: 10.29298/rmcf.v6i29.220

Raymond, C. A., Joe, B., Anderson, D W., \& Watt, D. J. (2008). Effect of thinning on relationships between three measures of wood stiffness in Pinus radiata: standing trees vs. logs vs. short clear specimens. Canadian Journal of Forest Research, 38(11), 2870-2879. doi: $10.1139 / \mathrm{X} 08-124$

Richards, F. J. (1959). A flexible growth function for empirical use. Journal of Experimental Botany, 10, 290-300.

Sakici, O. E., Misir, N., Yavuz, H., \& Misir, M. (2008). Stem taper functions for Abies nordmanniana subsp. bornmulleriana in Turkey. Scandinavian Journal of Forest Research, 23(6), 522-533. doi: $10.1080 / 02827580802552453$

Sánchez-González, M., Cardillo, E., Camacho E., Garriga, E., \& Cañellas, I. (2009). Modelo de crecimiento en diámetro para alcornoque en sistemas adehesados. En Sociedad Española de Ciencias Forestales (ed), $5^{\circ}$ Congreso Forestal Español (13 p). Ávila, España: SECF Junta de Castilla y León.

SAS Institute Inc. (2011). SAS/STAT® 9.3 User's Guide. Cary, NC USA: SAS Institute Inc.

Servicio y Consultoría Ambiental y Forestal [SCAF]. (2011). Estudio regional forestal Umafor 2101 - Izta Popo. Puebla, Puebla, México.

Sghaier, T., Palahi, M., Garchi, S., Bonet, J. A., Ammari, Y., \& Pique, M. (2012). Modeling dominant height growth in planted Pinus pinea stands in northwest of Tunisia. International Journal of Forestry Research, ID 902381, 12. doi: 10.1155/2012/902381

Sharma, R. P., Vacek, Z., Vacek, S., Jansa, V., \& Kučera, M. (2017). Modelling individual tree diameter growth for Norway spruce in the Czech Republic using a generalized algebraic difference approach. Journal of Forest Science, 63(5), 227-238. doi: 10.17221/135/2016-JFS

Vargas-Larreta, B., Álvarez-González, J. G., Corral-Rivas, J. J., \& Aguirre, C. Ó. A. (2010). Construcción de curvas dinámicas de índice de sitio para Pinus cooperi Blanco. Revista Fitotecnia Mexicana, 33(4), 343-351.

Vargas-Larreta, B., Aguirre-Calderón, O. A., Corral-Rivas, J. J., CrecenteCampo, F., \& Diéguez-Aranda, U. (2013). A dominant height 
growth and site index model for Pinus pseudostrobus Lindl. in northeastern Mexico. Agrociencia, 47(1), 91-106.

Wang, M., Borders, B. E., \& Zhao, D. (2008). An empirical comparison of two subject-specific approaches to dominant heights modeling: The dummy variable method and the mixed model method. Forest Ecology and Management, 255(7), 2659-2669. doi: 10.1016/j.foreco.2008.01.030

West, P. W., Ratkowsky, D. A., \& Davis, A. W. (1984). Problems of hypothesis testing of regressions with multiple measurements from individual sampling units. Forest Ecology and Management, 7(3), 207-224. doi:10.1016/0378-1127(84)90068-9

Xu, H., Sun, Y., Wang, X., Fu, Y., Dong, Y., \& Li, Y. (2014). Nonlinear mixed-effects (NLME) diameter growth models for individual china-fir (Cunninghamia lanceolata) trees in southeast China. PLoS ONE, 9(8), e104012. doi: 10.1371/journal.pone.0104012

Yang, R. C., Kozak, A., \& Smith, J. H. G. (1978). The potential of Weibull-type functions as flexible growth curves. Canadian Journal of Forest Research, 8(4), 424-431. doi: 10.1139/x78-062

Zimmerman, D. L., \& Núñez-Antón, V., Gregoire, T. G., Schabenberger, O., Hart, J. D., Kenward, M. G., \& Vieu, P.
(2001). Parametric modeling of growth curve data: an overview (with discussion). Test, 10: 1-73. doi: 10.1007/BF02595823

Manuscrito recibido el 30 de mayo de 2020.

Aceptado el 23 de febrero de 2021.

Publicado el 23 de diciembre de 2021

Este documento se debe citar como:

Tamarit-Urias, J. C., Quiñonez-Barraz, G., García-Cuevas, X., Hernández-Ramos, J., \& Monárrez-González, J. C. (2021). Ecuación dinámica para estimar el crecimiento en diámetro de Pinus montezumae Lamb. en Puebla, México. Madera y Bosques, 27(3), e2732180. doi: 10.21829/myb.2021.2732180

Madera y Bosques por Instituto de Ecología, A.C se distribuye bajo una Licencia Creative Commons Atribución-NoComercialCompartirlgual 4.0 Internacional. 